\title{
A Method to Discriminate Strain and Temperature in Fiber Bragg Grating Sensors
}

\author{
Madhan T Chalapathi, P K Pattnaik, A Selvarajan and T Srinivas \\ Department of Electrical Communication Engineering, \\ Indian Institute of Science, Bangalore, 560012, India \\ Phone: +91-80-229-33173, Fax: +91-80-236-00562, \\ Email: madhan_thollabandi@1ycos.com
}

\begin{abstract}
A significant limitation to the applications of fiber Bragg gratings (FBG) sensors is the sensitivity of the Bragg wavelength to both temperature and strain, complicating the independent measurement of these parameters. In this letter, we explore a new method to discriminate strain and temperature in FBG sensors, which utilizes the additional wavelength dependent phase introduced by Fiber Bragg grating written on sensing arm of Mach Zehnder Interferometer (MZI). The intensity variations of the MZI output at the wavelength outside the Bragg resonance provide an additional parameter along with the Bragg wavelength shift observed at the input end for simultaneous measurement of strain and temperature
\end{abstract}

\section{INTRODUCTION}

FIBER Bragg gratings sensors have generated great interest in recent years because of their potential use in a wide range of applications, particularly in monitoring the structural integrity of composite structures in aerospace applications. Fiber Bragg grating sensors provide absolute wavelength encoding of information, which depends upon strain and temperature effects acting on it. However, in general a single measurement of the Bragg wavelength shift cannot distinguish strain and temperature. Several discriminating techniques have been demonstrated in recent years to overcome this limitation. These include the use of dual wavelength superimposed gratings [1], hybrid Bragg grating/Long-period grating [2], dual-diameter FBGs [3], FBG superimposed with polarization-rocking filter [4], FBG combined with EDFA [5], FBG Fabry-Perot cavity method [6], superimposed fiber Bragg grating sensors [7], two Bragg gratings written on either side of a splice between fibers of dissimilar diameters [8] and super-structure FBGs (SFBG)[9]. Most of the techniques proposed uses two FBGs or single FBG with other elements. It is highly desirable to utilize a single sensing element to achieve discrimination between strain and temperature so that multiplexing of array of sensors can easily be achieved. Though the SFBG method employs a single SFBG to decouple the strain and temperature, it is difficult to fabricate and needs more careful selection of SFBG parameters to position the narrow-band loss peaks on the slope of broadband loss peak. In this letter, we demonstrate a new method to discriminate strain and temperature in fiber optic sensors by writing a Fiber Bragg grating on the sensing arm of the Mach-Zehnder interferometer.

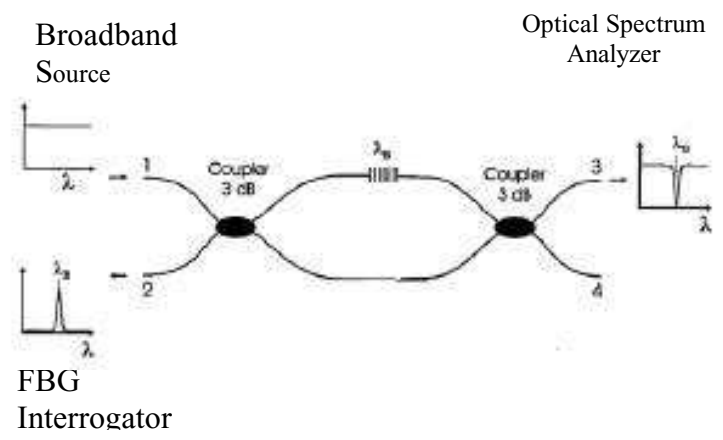

Fig. 1. Schematic of sensor configuration

\section{PRINCIPLE}

Figure 1 shows the schematic of the proposed sensor configuration. The sensor consists of a FBG written on sensing arm of a Mach-Zehnder interferometer formed by two $3 \mathrm{~dB}$ couplers. When light is launched from port 1 into the MZI, the grating will reflect light back to port 2 at Bragg wavelength. All the remaining wavelengths outside the Bragg resonance will undergo interference at port 3 . The intensity of light at the output for a particular wavelength will depend upon the phase difference between the two arms of MZI for that wavelength. The FBG in the sensing arm induces a wavelength dependent phase, which is a function of period and refractive index of the grating. Due to external perturbation, as the period and refractive index of the grating changes, the output intensity for an observation wavelength at port3 varies along with the Bragg wavelength shift at port 1 . The intensity variation at port 3 and the Bragg wavelength shift at port 1 can be used to simultaneously discriminate strain and temperature when acting together. The shift in Bragg peak $\left(\Delta \lambda_{B}\right)$ and change in intensity $(\Delta \mathrm{I})$ at MZI output can be expressed as a linear function of strain and temperature as follows.

$$
\begin{aligned}
& \Delta \lambda_{B}=\kappa_{11} \Delta \varepsilon+\kappa_{12} \Delta \mathrm{T} \\
& \Delta \mathrm{I}=\kappa_{21} \Delta \varepsilon+\kappa_{22} \Delta \mathrm{T}
\end{aligned}
$$

where $\kappa_{11}$ and $\kappa_{12}$ are the respective strain and temperature coefficients of the Bragg wavelength shift and $\kappa_{21}$ and $\kappa_{22}$ are strain and temperature coefficients of output intensity 
change at MZI. These coefficients can be found by applying known strain and temperatures separately on the sensor. We can estimate the unknown strain and temperature simultaneously by measuring the corresponding $\Delta \lambda_{B}$ and $\Delta I$ at an observation wavelength.

\section{THEORY}

Light from the narrowband source is coupled from port 1 into the arms of the balanced Mach-Zehnder interferometer by using a $3 \mathrm{~dB}$ coupler $\left(\mathrm{C}_{1}\right)$. For input light of electric field amplitude $\mathrm{A}_{0}$ at port 1 , the electric field amplitudes at the output of the first $3 \mathrm{~dB}$ coupler can be written as

$$
\begin{aligned}
& A_{1}=\frac{A_{0}}{\sqrt{2}} \\
& B_{1}=-j \frac{A_{0}}{\sqrt{2}}
\end{aligned}
$$

Since the light in the sensing arm experiences an additional phase delay of $\phi$ due to grating, the electric field amplitudes in the sensing arm and reference arm of the MZI at the coupler $\left(\mathrm{C}_{2}\right)$ input end $\mathrm{A}_{2}$ and $\mathrm{B}_{2}$ are given by,

$$
\begin{gathered}
A_{2}=\frac{A_{0}}{\sqrt{2}} \exp (-j \beta 1+j \phi) \\
B_{2}=\frac{B_{0}}{\sqrt{2}} \exp (-j \beta 1)
\end{gathered}
$$

The output amplitudes of the light at the port 3 and port 4 are given by,

$$
\begin{aligned}
& A_{3}=j A_{0} \exp \left(-j \beta 1+j \frac{\phi}{2}\right) \operatorname{Sin}\left(\frac{\phi}{2}\right) \\
& B_{3}=-j A_{0} \exp \left(-j \beta 1+j \frac{\phi}{2}\right) \operatorname{Cos}\left(\frac{\phi}{2}\right)
\end{aligned}
$$

where $\beta$ is the propagation constant and ' 1 ' is the length of the MZI arm. From the above equation, it is observed that the output of the MZI is a function of the phase delay induced by the FBG $(\phi)$ given by,

$$
\phi=\frac{2 \pi}{\lambda}\left(n_{\text {eff }}+\delta n\right) 1_{g}
$$

where $\delta n$ is the local refractive index change over the grating of length $l_{g}, \delta n$ is given by,

$$
\delta n(z)=\delta n_{0}\left(1+v \operatorname{Cos}\left(\frac{2 \pi z}{\Lambda}\right)\right)
$$

where $v$ and $\Lambda$ are the fringe visibility and period of the grating respectively and $\delta n_{0}$ is the average change in the refractive index of the grating.

When an external perturbation such as strain and temperature is applied to the grating on the sensing arm of MZI, the grating period alters and its refractive index changes giving rise to output intensity modulation. The output intensity can thus be written as

$$
A_{3}^{2}=-A_{0} \exp (-j 2 \beta 1+j \phi) \operatorname{Sin}^{2}\left(\frac{\phi}{2}\right)
$$

Where $\Delta \phi$ is given by,

$$
\begin{aligned}
\Delta \varphi=\int_{0}^{l_{g}} \frac{4 \pi}{\lambda}\left\{\left(n_{e f f} \Delta l_{g}+l_{g} \Delta n_{e f f}\right)+\left[1+\gamma \cos \left(\frac{2 \pi z}{\Lambda_{g}}\right)\right] \times\right. \\
{\left.\left[\Delta(\partial n) I_{g}+\partial n\left(\Delta l_{g}\right)+\gamma\left(\frac{2 \pi}{\Lambda_{g}^{2}}\right)(\partial n) \sin \left(\frac{2 \pi Z}{\Lambda_{g}}\right) \Delta \Lambda_{g}\right] d z\right\} }
\end{aligned}
$$

Change in grating period is given by,

$$
\Delta l_{g}=\Delta l_{g, \varepsilon}+\Delta l_{g, T}
$$

where $\Delta l_{g, \varepsilon}=\varepsilon l_{g}$ is change in grating length due to strain, $\varepsilon$ and $\Delta l_{g, T}=\alpha l_{g} \Delta T, \Delta T$ is change in grating length due to temperature $\mathrm{T}$ and $\alpha$ is the linear coefficient of thermal expansion of the fiber. Change in refractive index is given by,

$$
\Delta n=\Delta n_{\varepsilon}+\Delta n_{T}
$$

Where $\Delta n_{\varepsilon}=\rho_{\varepsilon} \varepsilon n$ is the change in refractive index due to elasto-optic effect and $\Delta n_{T}=\xi \Delta T n$ is change in refractive index due to temperature. $\rho_{\varepsilon}$ and $\xi$ are the strain-optic and thermo-optic coefficients of the fiber respectively. Due to external strain and temperature, the change in output intensity of MZI along with the Bragg wavelength shift can be used to discriminate the two as described in equation 1 and 2 .

\section{RESULTS AND DISCUSSION}

A fiber Bragg grating at $1545 \mathrm{~nm}$ Bragg wavelength was surface bonded on composite laminate $(25 \mathrm{~mm} \times 2.5 \mathrm{~mm} \times$ $0.25 \mathrm{~mm}$ ). This embedded FBG was connected in the sensing arm of the MZI formed by two $2 \times 23 \mathrm{~dB}$ couplers connected back to back keeping both the arms equal in length (figure.1). Light from tunable laser source of tuning range from 1520 $\mathrm{nm}$ to $1560 \mathrm{~nm}$ with repetition rate of $100 \mathrm{~Hz}$ was launched into port 1 . Tensile loads at $27{ }^{\circ} \mathrm{C}$ were applied on the composite laminate using a Zwick loading machine to get strain values upto $1000 \mu \varepsilon$. Due to this applied strain, the reflected Bragg wavelength peak at port 2 was measured using a laser spectra interrogator (Micron Optics). The intensity variations at the output port 3 for an observation wavelength of $1540 \mathrm{~nm}$ was continuously monitored for these applied strains. The coefficients $\kappa_{11}$ and $\kappa_{12}$ are obtained from the slopes of the plot Strain $(\varepsilon)$ Vs Intensity (I) and Temperature (T) Vs Intensity (I) respectively. Now, without applying any load, the laminate was subjected to temperature variations from $25{ }^{0} \mathrm{C}$ to $95{ }^{0} \mathrm{C}$. The reflected Bragg wavelength peak at port 2 and the intensity variations for an 
observation wavelength of $1530 \mathrm{~nm}$ at port 3 was measured. The coefficients $\kappa_{21}$ and $\kappa_{22}$ are obtained from the slopes of the plot Strain $(\varepsilon)$ Vs $\lambda_{B}$ and Temperature (T) Vs $\lambda_{B}$ respectively. Figure 2 shows the plot of Strain $(\varepsilon) \mathrm{Vs}$ intensity. We know that $\mathrm{K}_{11}=1 \mathrm{pm} / \mu$ strain and $\mathrm{K}_{21}=0.0035$ nw/ $\mu$ strain. Figure 3 shows the plot of Temperature (T) Vs Intensity (I). From the slopes, we estimate $\mathrm{K}_{12}=11.2 \mathrm{pm} /{ }^{0} \mathrm{C}$ and $\mathrm{K}_{22}=0.16 \mathrm{nw} /{ }^{\circ} \mathrm{C}$. Inserting the values of these coefficients into equation 1 and 2, unknown strain and temperature can simultaneously be measured.

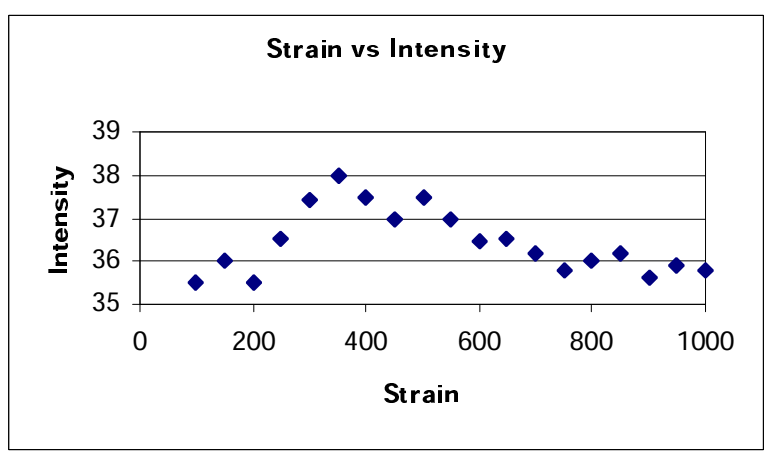

Fig. 2. Experimental results showing output intensity of the MZI as a function of strain.

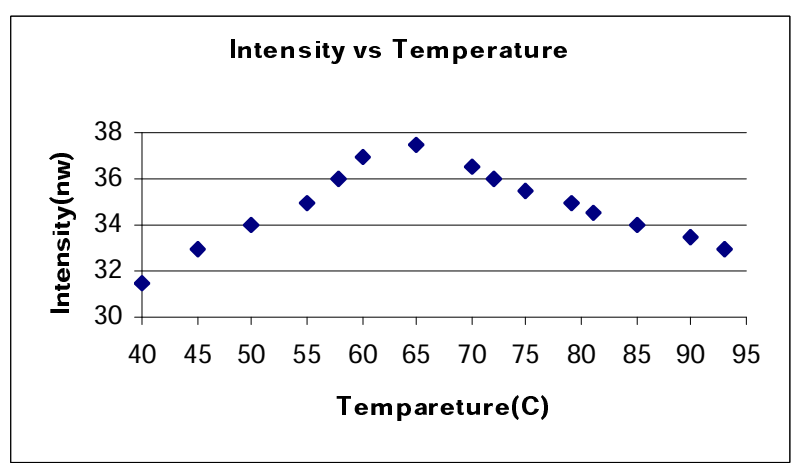

Fig. 3. Experimental results showing output intensity of the MZI as a function of Temperature.

\section{CONCLUSION}

We report the principle and experimental results of a novel sensor configuration that allows to simultaneously measure strain and temperature. This sensor has the advantages of being simple and used only single fiber Bragg grating. The experimental results show the feasibility of this sensor configuration. Multiplexing of array of sensors in this configuration need to be explored.

\section{ACKNOWLEDGMENT}

The authors wish to acknowledge Aeronautical Development Agency (ADA), Govt of India for supporting this work through DISMAS project. Also the authors wish to acknowledge H.V.Ramachandra, G.M.Kamath and Ramesh Sundaram, National Aerospace Laboratory for technical support. Also authors wish to acknowledge R. Usha, Department of Electronics, UVCE, Bangalore for useful discussion.

\section{REFERENCES}

[1] M.G.Xu, J.L.Archambault, L.Reekie, and J.P. Dakin, "Discrimination between strain and temperature effects using dual-wavelength fiber grating sensors", Electron Letters, vol. 30, pp. 1085-1087,1994.

[2] H.J.Patrick, G.M.Williams, A.D.Kersey, J.P.Pedrazzani, and A.M.Vengsarkar, "Hybrid fiber Bragg gratings/long period fiber grating sensor for strain/temperature discrimination", IEEE Photonics Technology Letters, vol. 8, no. 9, pp.1223-1225, 1996.

[3] S.W.James, M.L.Dockney, and R.P.Tatam, "Simultaneous independent temperature and strain measurement using in-fiber Bragg grating sensors", Electron Letters, vol. 32, no.12, pp.1133-1134, 1996.

[4] S.E.Kanellopoulos, V.A Handerek and A.J.Rogers, "Simultaneous strain and temperature sensing with photo generated in-fiber gratings", Optics Letters, vol.20, no.3, pp.333-335, 1995.

[5] J.Jung, H.Nam, J.H. Lee, N.Park, and B.Lee, "Simultaneous measurement of strain and temperature by use of a single-fiber Bragg grating and an erbium-doped fiber amplifier", Applied Optics., vol.38, no.13,pp.2749- 2751,1999.

[6] W.C.Du, X.M.Tao, and H.Y.Tam, "Fiber Bragg grating cavity sensor for simultaneous measurement of strain and temperature", IEEE Photonics Technology Letters, vol.11, no.1, pp.105-107, 1999.

[7] B.J.Eggleton, P.A.Krug, L.Poladian and F.Ouellette, "Long periodic super-structure Bragg gratings in optical fibers”, Electron Letters, vol. 30, no.19, pp. 1620-1622, 1994.

[8] V.Bhatia and A.M. Vengsarkar, "Optical fiber long period grating sensors", Optics Letters, vol. 21, no.9, pp. 692-694, 1996.

[9] Bai-Ou Guan, Hwa-Yaw, Xiao-Ming Tao and Xiao-Yi Dong, "Simultaneous strain and temperature measurement using a superstructure fiber Bragg grating", IEEE Photonics Technology Letters., vol.12, no.6,pp.675-677,2000. 